\title{
A review on milk allergy
}

\section{Abstract}

Milk allergy, sometimes synonymously used with lactose intolerance, is one of the most common allergies in children and adults. It is a true food allergy caused by an allergic reaction to the protein in milk and its products. ${ }^{1}$ Milk allergy is one of the common allergies in children and babies, while lactose intolerance is common in adults. Milk allergy is very much different from lactose intolerance, which is non-allergic food sensitivity, due to the lack of enzyme lactase in the small intestines to break lactose down into glucose and galactose. ${ }^{2}$

Keywords: lactose intolerance, anaphylaxis, casein, whey protein
Volume 8 Issue I - 2018

\section{Zainab Hussain}

Food Service Dietitian at Safe Food Caterers (SFC), Tabba Heart Institute (THI), Pakistan

\begin{abstract}
Correspondence: Zainab Hussain, BS (Nutrition \& Dietetics) CRCP, Food Service Dietitian at Safe Food Caterers (SFC), Tabba Heart Institute (THI), Web Committee Chair, Pakistan Nutrition and Dietetic Society (PNDS), Pakistan, Email zainabhussain874@gmail.com
\end{abstract}

Received: January 25, 2018 | Published: February 20, 2018

\section{Introduction}

\section{Milk allergy}

Milk or dairy allergy involves the immune system. Dairy allergic person's body reacts to proteins in milk and other dairy products as if they are dangerous invaders. It releases substances that cause allergy symptoms. This allergic reaction can be mild (rashes) too severe (trouble breathing, loss of consciousness) (Figure 1).

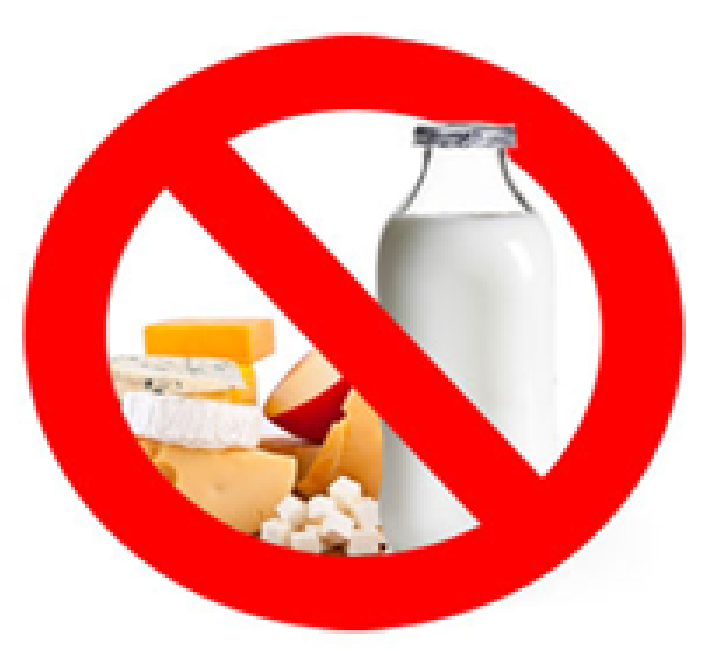

Figure I lactose intolerance.

\section{Difference between milk allergy and lactose intoleran- ce}

Where milk allergy refers to the involvement of immune system, on the contrary, lactose intolerance is related to digestive system. It caused by not having enough of the enzyme lactase, which is needed to break down lactose, the sugar found in milk and other dairy products.

Lactose is one of the disaccharides contained within the brush border of small intestine epithelial cells. In the human, lactase activity is detectable in the fetal gut as early as 8weeks' gestation. Persons who have a deficiency of the intestinal enzyme lactase have a decreased ability to digest lactose and experience symptoms of abdominal cramping and etc. Others may not lack intestinal lactase activity, lactose is absorbed and marked lactosuria together with aminoaciduria, proteinuria and renal tubular acidosis is found. ${ }^{1}$ Infants may develop severe diarrhea, vomiting and acidosis within the first few days of life and is a life threatening situation. It should be noted that even a small amount of lactose can precipitate the symptoms, but it is a transient disorder from which the child recovers between 12 and 18 months of age.

\section{Prevalence of milk allergy}

It is observed that milk allergy is most common in Asians at approximately $90 \%$ of the total food allergic population (Figure 2).

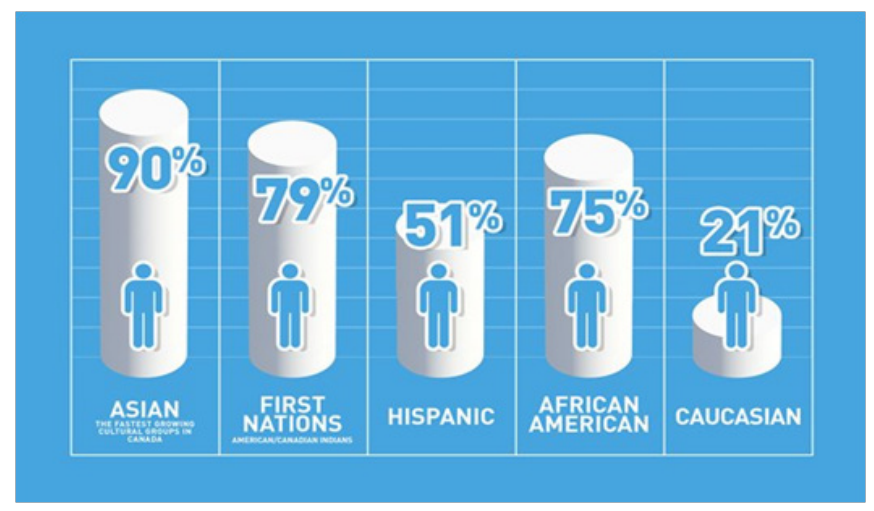

Figure 2 Prevalence of milk allergy.

\section{Kinds of milk allergy}

A person with a milk allergy will often be allergic to one of two main proteins in milk: casein or whey.

Casein allergy: Casein is a protein found in milk and other dairy products. A casein allergy occurs when your body mistakenly identifies casein as a threat itself. The body then triggers a reaction in an attempt to fight it off and allergic symptoms appear. Infants who are breast-fed are at a lower risk of developing a casein allergy. Usually, a casein allergy will go away by the time a child reaches 3 to 5 years of age. Some children never outgrow their casein allergy and may have it into adulthood. ${ }^{3}$ 
Whey allergy: Another milk protein associated with food allergies is whey. Whey protein is a derivative of milk, so one may experience allergic symptoms associated with a dairy allergy. Not all side effects from whey protein are related to allergies, however; some side effects stem from intolerance as well. Sometimes whey is blended with other protein powders or is hidden in high-protein bars or drinks so it is important for persons with whey allergy to opt for the substitutes very vigilantly. ${ }^{3}$

\section{Types of milk allergy}

People with regular milk allergy may be allergic to other kinds of milk as well like almond milk, rice milk or soy milk.

Almond milk allergies: Switching from regular milk to almond milk may not be a good option for some people as it is only trading one allergic reaction to another. This means that people allergic to regular milk are likely to be allergic to almonds also. Tree nuts such as almonds (along with walnuts, cashews, and pecans) top the list of allergy offenders. Nearly half of people allergic to milk may observe allergy to almond milk as well. Unlike a cow's milk allergy, which typically resolves at a very early age, blend of milk and almond allergies tend to last a lifetime.

Rice milk allergies: Rice is the least likely grain to cause an allergic reaction. Many parents, after their child is diagnosed with regular milk allergy, choose to give their children rice milk instead of cow's milk. But some may not accept this substitute as well. However, rice allergies are extremely rare in the West; they have been on the rise in Asian countries, where rice is a staple food.

Soy milk allergies: Soy is one of the "big eight" allergens, so it's important to watch for symptoms, especially in children. Regular milk allergic children are often allergic to soy; therefore the parents should choose the substitutes wisely. Soybeans, along with peanuts, kidney beans, lentils, and peas, are in the legume family and may trigger an allergic reaction.

\section{Symptoms of milk allergy}

Milk allergies can have fast onset (from seconds tohours) or slow onset (fromhours todays). Symptoms may include: rash, hives, itching, swelling, difficulty swallowing, runny or congested nose, wheezing, shortness of breath, diarrhea, nausea and vomiting. When these symptoms occur, the allergic reaction is usually called anaphylaxis.

\section{Diagnosing milk allergy}

Milk allergy can be diagnosed by the person's history of allergic reactions, skin prick test (SPT), patch test and measurement of milk protein specific serum immunoglobulin E (IgE or SIgE). Confirmation of the allergy can be done by double-blind, placebo-controlled food challenges, conducted by an allergy specialist. ${ }^{4} \mathrm{~A}$ skin prick test, sometimes called a puncture or scratch test, is used to check for immediate allergic reactions. This test is usually done to identify allergies to pollen, different foods etc. In adults, the test is usually done on the forearm. They sometimes tell erroneous results, telling that you are not allergic to certain food, even if you are, that is why one should not completely rely on those results (Figure 3 ). ${ }^{5}$

\section{Consequences of milk allergy}

Children with different food allergies usually have poor growth pattern as compared to non-allergic children because of the elimination of some products from their diet. Therefore, it is very important to give more attention to the diets of such children to help them in growing properly according to their age, gender and nutrient needs. It is also important to keep a regular follow up of these children. The periodic re-evaluation of the child is needed to assess the nutritional needs, changing with the age, and the compliance to the diet. The follow- up plan should be established on the basis of the age of the child and following the growth pattern.

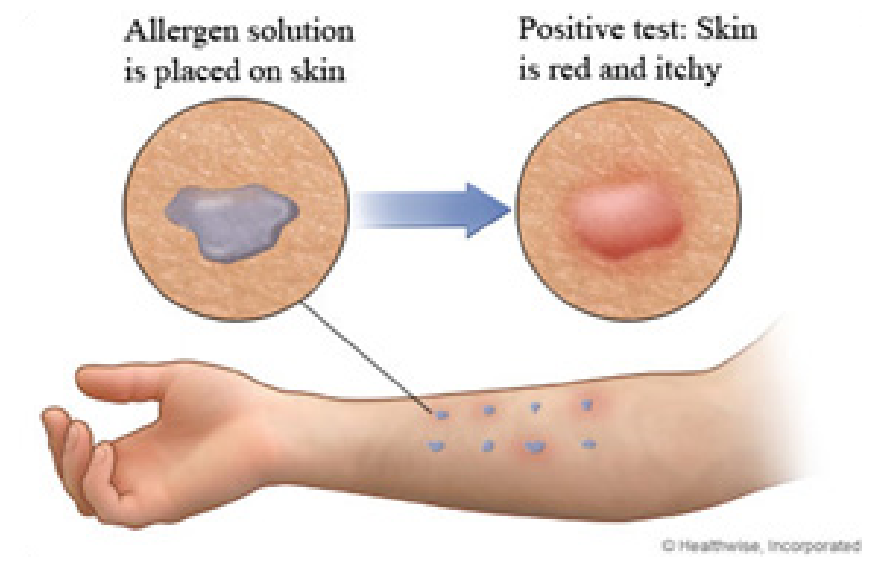

Figure 3 Skin prick test.

\section{Foods to avoid}

The following foods and ingredients DO NOT contain milk protein and can be eaten:

Cocoa butter, Cream of tartar, Lactic acid, sodium or calcium lactate, Sodium or calcium stearoyl lactylate. ${ }^{6}$

\section{Substitutions to some foods:}

a. Instead of cow's milk infant formula for a child less than 1year of age, breast milk or appropriate formula as advised by your child's medical specialist can be substituted.

b. Yoghurt can be substituted with soy yoghurt.

c. Cheese and sour cream can be substituted with soy cheese or soy sour cream.

d. Instead of ice cream, soy ice cream, sorbet, milk free gelato can be used.

e. Butter and margarine Oil can be substituted with milk free margarine e.g. Nuttelex.

f. Instead of cream and condensed milk, soy cream, soy cooking milk, soy condensed milk can be used.

g. Rice cream can be substituted with chocolate soy or rice based (milk free) chocolate. ${ }^{6}$

\section{Formula for babies with milk allergies}

Most pediatricians recommend soy-based formulas with added vitamins and minerals for babies allergic to milk. ${ }^{7}$ If symptoms don't improve after a switch to soy, hypoallergenic formulas are available. These include extensively hydrolyzed formulas in which proteins have been broken down so they are less likely to cause a reaction. The other type of hypoallergenic formula commonly used is known as an elemental formula, in which only the simplest forms of protein are used. 


\section{Discussion}

\section{Role of diet in the management of milk allergy}

The cornerstone of the nutritional management of food allergies is an individualized allergen avoidance management plan. In children, the main goals are to prevent the occurrence of acute and chronic symptoms by avoiding the offending food(s), whilst providing an adequate, healthy and nutritionally balanced diet and maintaining optimal growth; ideally, under the guidance of a trained dietitian. Complete avoidance of the allergen is still required by some, but latest developments in food allergy have indicated that some individuals with food allergies tolerate baked forms of milk.

It is known that children and families with food allergies experience a decreased quality of life across a number of domains, which can create anxiety and lead to avoidance of social situations. Hence, it is suggested that liberalization of the diet, when appropriate and safe, will increase both quality of life and nutritional intake. ${ }^{8}$

\section{The dietitian's role in food allergy management}

When your child is first diagnosed with food allergies, what is the first thing you do? You think, "What will I feed my child? How will I make sure I provide foods that are nutritious but won't trigger an allergic reaction?"

A dietitian may be the first person who comes to mind to answer this question. Perhaps your allergist recommended a dietitian to help you plan an allergen-free diet.

\section{How a dietitian can help your child}

During a visit with a dietitian, she will discuss your child's normal intake. Also, you will discuss symptoms that have occurred with the ingestion of suspected foods. You should compare the nutritional needs of your child with current intake. This will help to determine if more nutrition-dense foods or supplements are necessary. You should outline a meal plan. The nutritionist should provide you with food/ symptom diaries and a list of foods to avoid. He/she should give you suggestions for meals including which foods to use. Make sure to schedule a follow-up visit. The follow-up visit allows you to discuss progress, as well as concerns. You may also need to make an alternate plan for suggestions that did not work. ${ }^{9}$

The role of the dietitian differs between countries depending on the extent of the physician's role in the dietary management. In some countries the dietitians are trained to be involved in the diagnosis, while in other countries the dietitians are involved in the dietary management only. ${ }^{10}$

Nutrition plays a key role in the development, maintenance, and optimal functioning of immune cells. Nutrients, such as zinc and vitamin $\mathrm{D}$ and nutritional factors, such as pre- and probiotics, can influence the nature of an immune response and are important in ensuring appropriate functioning of the immune system. ${ }^{8}$

\section{Acknowledgements}

The author would like to thank Mr Mateen Anwer for helping her in reviewing this article.

\section{Conflict of interest}

Author declares that there is no conflict of interest.

\section{References}

1. Hussain Z. Food Allergy. Journal of Immunology and Infectious Diseases. 2018;4(2):1-5.

2. Heine RG, AlRefaee F, Bachina $\mathrm{P}$, et al. Lactose intolerance and gastrointestinal cow's milk allergy in infants and children - common misconceptions revisited. World Allergy Organ J. 2017;10(1):41.

3. Casein Allergy Information: Causes, Diagnosis, and Risks. Healthline. 2018.

4. Soares-Weiser K, Takwoingi Y, Panesar SS, et al. The diagnosis of food allergy: a systematic review and meta-analysis. Allergy. 2014;69(1):76-86.

5. Allergy skin tests. Mayo Clinic. 2018.

6. Dietary avoidance cow's milk protein (dairy) allergy. 2015.

7. Milk Allergies (Milk Protein Allergy). Healthline. 2017.

8. Mazzocchi A, Venter C, Maslin K, et al. The Role of Nutritional Aspects in Food Allergy: Prevention and Management. Nutrients. 2017;9(8).

9. Plus G. How a Dietitian Can Help with Food Allergies. 2018.

10. Venter C, Laitinen K, Vlieg-Boerstra B. Nutritional aspects in diagnosis and management of food hypersensitivity-the dietitians role. J Allergy (Cairo). 2012; 2012:269376. 